\title{
Calculation of the potential of mean force from nonequilibrium measurements via maximum likelihood estimators
}

\author{
Riccardo Chelli, Simone Marsili, Piero Procacci \\ Dipartimento di Chimica, Università di Firenze, \\ Via della Lastruccia 3, I-50019 Sesto Fiorentino, Italy and \\ European Laboratory for Non-linear Spectroscopy (LENS), \\ Via Nello Carrara 1, I-50019 Sesto Fiorentino, Italy
}

(Dated: October 25, 2018)

\begin{abstract}
We present an approach to the estimate of the potential of mean force along a generic reaction coordinate based on maximum likelihood methods and path-ensemble averages in systems driven far from equilibrium. Following similar arguments, various free energy estimators can be recovered, all providing comparable computational accuracy. The method, applied to the unfolding process of the $\alpha$-helix form of an alanine deca-peptide, gives results in good agreement with thermodynamic integration.
\end{abstract}




\section{INTRODUCTION}

Estimate of free energy differences is useful for many applications including protein/ligand binding affinities and drug design as well as for theoretical perspectives. A rough classification of the plethora of computational methods devised for determining free energy differences can be based on the possibility of sampling a system at equilibrium or out of equilibrium. Equilibrium approaches include thermodynamic integration[1], free energy perturbation[2] and Umbrella Sampling techniques [3]. Representative examples of nonequilibrium techniques are the so-called adaptive force [4] or potential [5] bias methods. The efficiency of the latter techniques depends crucially on how fast the historydependent force or potential changes in time, or in other words, how far from equilibrium the simulation is carried out. From this point of view, adaptive bias potential methods would be more appropriately defined as quasi-equilibrium techniques.

In the context of nonequilibrium approaches [6, 7], a substantially different scenario has been disclosed by Jarzynski [8] and Crooks [9], who introduced "truly" nonequilibrium methods for determining free energy differences. In particular they proposed two exact equations, referred here as Jarzynski equality and Crooks nonequilibrium work theorem, relating free energy differences between two thermodynamic states to the external work done on the system in an ensemble of nonequilibrium paths switching between the two states. In a recent paper Shirts et al. [10] have demonstrated that the Bennett acceptance ratio 11 can be interpreted, exploiting the Crooks nonequilibrium work theorem, in terms of the maximum likelihood (ML) estimate of the free energy difference given a set of nonequilibrium work values in the forward and reverse directions.

One of the major shortcomings of these nonequilibrium techniques is that free energy profile along a given reaction coordinate, i.e. the potential of mean force (PMF), is hardly available. With the two sets of forward and reverse nonequilibrium paths, Crooks nonequilibrium work theorem [9] and ML method[10] yield only the free energy differences between the final and initial states. The Jarzynski equality, on the other hand, can in principle be used to calculate the PMF. However it is well-known [12, 13, 14, 15] that the exponential average in the Jarzynski equality depends crucially on a small fraction of realizations that transiently violate the second law of thermodynamics. Since such "magic" realizations are very unlikely to occur among a collection of fast rate realizations, it is clear that the potential of mean force cannot be determined accurately by the direct application of the Jarzynski equality.

In the present paper we demonstrate how to recover the PMF using ML estimators [10] and path-ensemble averages in systems driven far from equilibrium[16]. We test the method on the unfolding process of the $\alpha$-helix form of an alanine deca-peptide through steered molecular dynamics (MD) simulations.

\section{THEORY}

\section{A. Description of the dynamical system and notation}

Let us consider a system that can switch between two states, $A$ and $B$, characterized by different values of an arbitrary reaction coordinate $\zeta$, namely $\zeta_{A}$ and $\zeta_{B}$. We denote with $F$ (forward) any realization during which the reaction coordinate is forced to vary from $\zeta_{A}$ to $\zeta_{B}$ with a prescribed time schedule. Accordingly, we denote with $R$ (reverse) any realization that brings the reaction coordinate from $\zeta_{B}$ to $\zeta_{A}$ with inverted time schedule. The kind of computational or experimental technique used for producing the realizations is not relevant. The essential requirement is that the used technique furnishes the value of the work done on the system during the realizations. Suppose to produce a collection of $n_{F}$ forward realizations and a collection of $n_{R}$ reverse realizations, each realization being started from microstates (i.e., phase space points) sampled from an equilibrium distribution (equilibrium microstates of $A$ for the $F$ realizations and equilibrium microstates of $B$ for the $R$ realizations). Specifically, an equilibrium microstate of, e.g., $A$ is simply obtained by sampling the system in thermal equilibrium with a bath, the reaction coordinate being constrained to the value $\zeta_{A}[13,17]$. Furthermore, we assume that all realizations are performed at a very fast rate, which implies that they are carried out far from equilibrium. As a consequence, the final microstates of the $F$ and $R$ realizations will not be distributed according to the equilibrium distribution of $B$ and $A$, respectively. It is evident that the same holds true for the intermediate microstates of the $F$ and $R$ realizations. For example, the microstates characterized by a generic value $\zeta_{Q}$ of the reaction coordinate obtained during a realization starting either from $A$ ( $F$ realization) or from $B$ ( $R$ realization) will not be equilibrium microstates of the state $Q$ characterized by the reaction coordinate $\zeta_{Q}$. This situation is schematically represented in Fig. 1, We now denote a generic $i$ th $F$ realization with $\mathbf{F}_{i}^{A b}$, where the superscript $A b$ means that the thermodynamic state corresponding to the initial microstate is $A$ (first letter) and that such microstate is taken from an equilibrium ensemble of microstates (uppercase), while the thermodynamic state corresponding to the final microstate is $B$ (second letter) and that such microstate belongs to an ensemble of microstates out of equilibrium (lowercase). Following this notation, the segments from $\zeta_{A}$ to $\zeta_{Q}$ and 
from $\zeta_{Q}$ to $\zeta_{B}$ of the $\mathbf{F}_{i}^{A b}$ realization are denoted as $\mathbf{F}_{i}^{A q}$ and $\mathbf{F}_{i}^{q b}$, such that $\mathbf{F}_{i}^{A b} \equiv \mathbf{F}_{i}^{A q}+\mathbf{F}_{i}^{q b}$. Analogously, we may write $\mathbf{R}_{j}^{B a} \equiv \mathbf{R}_{j}^{B q}+\mathbf{R}_{j}^{q a}$. The same symbols with no specified subscripts will be used to indicate a generic realization or a collection of realizations. Finally, we define the free energy difference between the states $A$ and $B$ as $\Delta F_{A B}=F_{B}-F_{A}$.

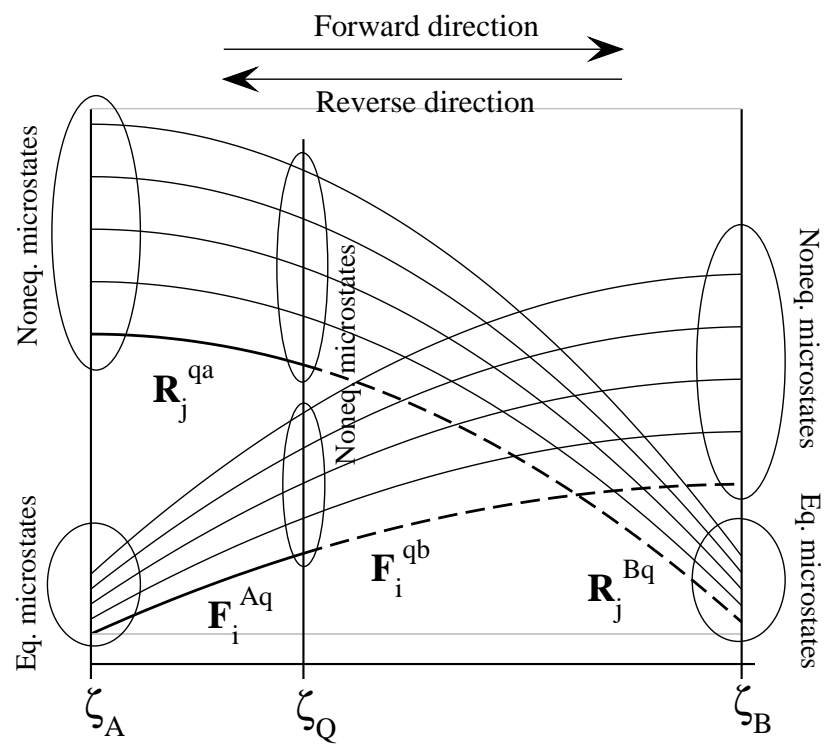

FIG. 1: Schematic representation of forward and reverse realizations with the notation used in the text.

\section{B. Background}

Given these two collections of nonequilibrium realizations, one may recover $\Delta F_{A B}$ following the ML method by Shirts et al.[10]. Such method is based on the maximization of the overall likelihood of obtaining the series of measurements (specifically the work done on the system during the $F$ and $R$ realizations) using the free energy difference as variational parameter. In our case, the likelihood $\mathcal{L}$ of obtaining the given work measurements can be expressed as the joint probability of obtaining the forward measurements at the specified work values $W\left[\mathbf{F}_{1}^{A b}\right]$, $W\left[\mathbf{F}_{2}^{A b}\right], \ldots, W\left[\mathbf{F}_{n_{F}}^{A b}\right]$, times the joint probability of obtaining the reverse measurements at the specified work values $W\left[\mathbf{R}_{1}^{B a}\right], W\left[\mathbf{R}_{2}^{B a}\right], \ldots, W\left[\mathbf{R}_{n_{R}}^{B a}\right]:$

$$
\mathcal{L}\left(\Delta F_{A B}\right)=\prod_{i=1}^{n_{F}} P\left(F \mid W\left[\mathbf{F}_{i}^{A b}\right]\right) \prod_{j=1}^{n_{R}} P\left(R \mid W\left[\mathbf{R}_{j}^{B a}\right]\right),
$$

where $W\left[\mathbf{F}_{i}^{A b}\right]$ and $W\left[\mathbf{R}_{j}^{B a}\right]$ are the work performed on the system during the $\mathbf{F}_{i}^{A b}$ and $\mathbf{R}_{j}^{B a}$ realizations. The best estimate of the free energy difference $\Delta F_{A B}$ is the value that maximizes $\mathcal{L}\left(\Delta F_{A B}\right)$, or equivalently its $\log$ function:

$$
\frac{\partial \ln \mathcal{L}\left(\Delta F_{A B}\right)}{\partial \Delta F_{A B}}=\sum_{i=1}^{n_{F}} \frac{1}{1+\frac{n_{F}}{n_{R}} \mathrm{e}^{\beta\left(W\left[\mathbf{F}_{i}^{A b}\right]-\Delta F_{A B}\right)}}-\sum_{j=1}^{n_{R}} \frac{1}{1+\frac{n_{R}}{n_{F}} \mathrm{e}^{\beta\left(W\left[\mathbf{R}_{j}^{B a}\right]+\Delta F_{A B}\right)}}=0,
$$

where $\beta=\left(k_{B} T\right)^{-1}, k_{B}$ being the Boltzmann constant and $T$ the temperature. A full derivation of above equation can be found in Ref. 10. We point out that Eq. 2 has been derived starting from the Crooks nonequilibrium work theorem [9, 16]. This implies that the time schedules of the $F$ and $R$ realizations must be related by time reversal symmetry and that the initial microstates of the realizations must be sampled from equilibrium distributions. Note also that Eq. 2 is exactly equivalent to the Bennett acceptance ratio method, as can be seen by comparison to Eqs. $12(\mathrm{a})$ and $12(\mathrm{~b})$ of Ref. 11. 


\section{Central result}

Suppose we want to determine the free energy difference $\Delta F_{A Q}$ between the states $A$ and $Q$ using the $F$ and $R$ realizations introduced above. We recall that $Q$ is an intermediate thermodynamic state between $A$ and $B$, in the sense that it is characterized by a reaction coordinate, $\zeta_{Q}$, which is taken arbitrarily from the path connecting $\zeta_{A}$ to $\zeta_{B}$, or viceversa (see Fig. 11). As explained above, this free energy difference cannot be determined simply exploiting our collections of $F$ and $R$ realizations into Eq. 2 because the segments of the $R$ realizations generally indicated as $\mathbf{R}^{q a}$ do not start from equilibrium microstates of the state $Q$. However, had the $R$ realizations been started from equilibrium microstates of $Q$, i.e., suppose that the $\mathbf{R}^{Q a}$ realizations are available in the place of the $\mathbf{R}^{q a}$ ones, then we could apply Eq. 2 for the calculation of $\Delta F_{A Q}$. In the resulting equation, which is equivalent to Eq. 2 with $\mathbf{F}_{i}^{A b}$, $\mathbf{R}_{j}^{B a}$ and $\Delta F_{A B}$ replaced by $\mathbf{F}_{i}^{A q}, \mathbf{R}_{j}^{Q a}$ and $\Delta F_{A Q}$, respectively, the second sum can be rearranged as follows

$$
\sum_{i=1}^{n_{F}} \frac{1}{1+\frac{n_{F}}{n_{R}} \mathrm{e}^{\beta\left(W\left[\mathbf{F}_{i}^{A q}\right]-\Delta F_{A Q}\right)}}-\int_{-\infty}^{+\infty} \frac{n_{R}\left\langle\delta\left(W\left[\mathbf{R}^{Q a}\right]-W\right)\right\rangle}{1+\frac{n_{R}}{n_{F}} \mathrm{e}^{\beta\left(W+\Delta F_{A Q}\right)}} \mathrm{d} W=0,
$$

where $\delta$ is the Dirac delta function and $\left\langle\delta\left(W\left[\mathbf{R}^{Q a}\right]-W\right)\right\rangle$ is a shorthand for $n_{R}^{-1} \sum_{j=1}^{n_{R}} \delta\left(W\left[\mathbf{R}_{j}^{Q a}\right]-W\right)$. We stress again that the initial microstates of the $\mathbf{R}^{Q a}$ realizations are assumed to be sampled from equilibrium. Of course, since we are dealing with nonequilibrium $\mathbf{R}^{B a}$ realizations, work measurements $W\left[\mathbf{R}^{Q a}\right]$ are unavailable, at least directly. Thus, the basic problem here is to derive the unknown quantity $\left\langle\delta\left(W\left[\mathbf{R}^{Q a}\right]-W\right)\right\rangle$ using somehow the overall physical information contained into our $\mathbf{R}^{B a}$ realizations.

To this aim we take advantage of a relation due to Crooks [16] that establishes a correlation between a function of the microstate of the system determined along forward and reverse realizations and the dissipated work done on the system during either the forward or the reverse realizations. In particular, setting $f[x]$ to be a function of the final microstate $x$ of a forward realization and $f[\hat{x}]$ to be the same function of the initial microstate $\hat{x}$ of a reverse realization, the following relation holds:

$$
\langle f[\hat{x}]\rangle_{R}=\left\langle f[x] \mathrm{e}^{-\beta W_{d}}\right\rangle_{F},
$$

where $W_{d}$ is the work dissipated during the $F$ realization. The subscripts $F$ and $R$ indicate that the ensemble averages are calculated on collections of forward and reverse realizations, respectively. Therefore, since the average $\langle f[\hat{x}]\rangle_{R}$ is computed on the initial (equilibrium) ensemble of the reverse process, the subsequent dynamics of the system is irrelevant and the average equals an equilibrium average of the function $f[\hat{x}]$. In applying Eq. 4 to our case, we consider the $\mathbf{R}^{B q}$ realizations (see Fig. 1) as the forward ones. This implies that the left side of Eq. 4 refers to an ensemble average of the equilibrium state $Q$. Moreover, for a given microstate of the system $x_{i}$, corresponding to the final microstate of the $\mathbf{R}_{i}^{B q}$ realization, we set

$$
f\left[x_{i}\right]=\delta\left(W\left[\mathbf{R}_{i}^{q a}\right]-W\right),
$$

where $W$ is an arbitrary real number and $\mathbf{R}_{i}^{q a}$ is a segment of the $\mathbf{R}_{i}^{B a}$ realization. We remark that, given a deterministic dynamical system and given a time schedule for evolving the reaction coordinate, the quantity $\delta\left(W\left[\mathbf{R}_{i}^{q a}\right]-W\right)$ is a single value function of the microstate $x_{i}$. With this provision, the general Eq. 4 takes the following specific form

$$
\left\langle\delta\left(W\left[\mathbf{R}^{Q a}\right]-W\right)\right\rangle=\left\langle\delta\left(W\left[\mathbf{R}^{q a}\right]-W\right) \mathrm{e}^{-\beta W_{d}\left[\mathbf{R}^{B q}\right]}\right\rangle,
$$

where $W_{d}\left[\mathbf{R}^{B q}\right]$ is the work dissipated in the $\mathbf{R}^{B q}$ realizations. Since $\Delta F_{B Q}$ is unknown, $W_{d}\left[\mathbf{R}^{B q}\right]$ cannot be determined. However, upon division of Eq. 6 by the equality [16] $\left\langle\exp \left(-\beta W_{d}\left[\mathbf{R}^{B q}\right]\right)\right\rangle=1$, and using the definition $W_{d}\left[\mathbf{R}_{i}^{B q}\right]=W\left[\mathbf{R}_{i}^{B q}\right]-\Delta F_{B Q}$, we obtain

$$
\left\langle\delta\left(W\left[\mathbf{R}^{Q a}\right]-W\right)\right\rangle=\frac{\left\langle\delta\left(W\left[\mathbf{R}^{q a}\right]-W\right) \mathrm{e}^{-\beta W\left[\mathbf{R}^{B q}\right]}\right\rangle}{\left\langle\mathrm{e}^{-\beta W\left[\mathbf{R}^{B q}\right]}\right\rangle} .
$$

This equation states that the distribution of the work $W\left[\mathbf{R}^{Q a}\right]$ done on the system in a collection of nonequilibrium realizations switching the reaction coordinate from $\zeta_{Q}$ to $\zeta_{A}$ and starting from equilibrium microstates can be recovered from a set of nonequilibrium realizations switching the reaction coordinate between the same values, but starting from nonequilibrium microstates (realizations $\mathbf{R}^{q a}$ in Eq. 7). The contribution of each work measurement $W\left[\mathbf{R}^{q a}\right]$ to the distribution must however be weighted by a factor depending on the work done on the system to produce the initial 
nonequilibrium microstate (the factor $\exp \left(-\beta W\left[\mathbf{R}_{i}^{B q}\right]\right) /\left\langle\exp \left(-\beta W\left[\mathbf{R}^{B q}\right]\right)\right\rangle$ in Eq. 7). We point out that, while Eq. 4 is valid for both stochastic and deterministic systems [16], the derivation of Eq. 7 provided here holds only for deterministic systems (we have indeed introduced this assumption when defining $f\left[x_{i}\right]$; see Eq. 5). However, it has been numerically proved[21] that the relations derived in the present article (specifically Eq. 16) can also be applied successfully to Brownian dynamical systems. Finally, substituting Eq. 7 into Eq. 3 and performing the integral, we get

$$
\sum_{i=1}^{n_{F}} \frac{1}{1+\frac{n_{F}}{n_{R}} \mathrm{e}^{\beta\left(W\left[\mathbf{F}_{i}^{A q}\right]-\Delta F_{A Q}\right)}}-\left\langle\mathrm{e}^{-\beta W\left[\mathbf{R}^{B q}\right]}\right\rangle^{-1} \sum_{j=1}^{n_{R}} \frac{\mathrm{e}^{-\beta W\left[\mathbf{R}_{j}^{B q}\right]}}{1+\frac{n_{R}}{n_{F}} \mathrm{e}^{\beta\left(W\left[\mathbf{R}_{j}^{q a}\right]+\Delta F_{A Q}\right)}}=0 .
$$

The above equation is the central result of the present article. By means of a recursive procedure, Eq. 8 allows us to determine the free energy difference between the state $A$ and an arbitrary state $Q$, and hence between any pair of states along the reaction path. We note that in Eq. 8 the physical information of both $F$ and $R$ realizations is used, albeit not at the maximum extent. In fact, while the $R$ realizations are fully used (note that $\mathbf{R}_{j}^{B q}+\mathbf{R}_{j}^{q a} \equiv \mathbf{R}_{j}^{B a}$ ), for the $F$ realizations only the segments $\mathbf{F}^{A q}$ are actually employed.

An analogous and symmetrical approach aimed at using the physical information contained into the full $\mathbf{F}^{A b}$ realizations and into the segment $\mathbf{R}^{B q}$ of the $\mathbf{R}^{B a}$ realizations allows us to recover a ML estimator to determine $\Delta F_{Q B}:$

$$
\left\langle\mathrm{e}^{-\beta W\left[\mathbf{F}^{A q}\right]}\right\rangle^{-1} \sum_{i=1}^{n_{F}} \frac{\mathrm{e}^{-\beta W\left[\mathbf{F}_{i}^{A q}\right]}}{1+\frac{n_{F}}{n_{R}} \mathrm{e}^{\beta\left(W\left[\mathbf{F}_{i}^{q b}\right]-\Delta F_{Q B}\right)}}-\sum_{j=1}^{n_{R}} \frac{1}{1+\frac{n_{R}}{n_{F}} \mathrm{e}^{\beta\left(W\left[\mathbf{R}_{j}^{B q}\right]+\Delta F_{Q B}\right)}}=0 .
$$

As for Eq. 2, the left sides of Eqs. 8 and 9 are strictly increasing functions of $\Delta F_{A Q}$ and $\Delta F_{Q B}$, respectively. The limits of the left side of Eq. 8 for $\Delta F_{A Q} \rightarrow \infty$ and for $\Delta F_{A Q} \rightarrow-\infty$ are $n_{F}$ and $-n_{R}$, respectively. The analogous limits of the left side of Eq. 9, i.e., $\Delta F_{Q B} \rightarrow \infty$ and $\Delta F_{Q B} \rightarrow-\infty$, give the same values. The monotonic behavior of the left sides of Eqs. 8 and 9 and their limit values guarantee the existence of one unique root. Such root corresponds to the value of the free energy difference that furnishes the ML estimate of the measured/calculated data. It is finally straightforward to prove that both equations have Eq. 2 as special case (set the equivalence between the states $Q$ and $B$ in Eq. 8 and between the states $Q$ and $A$ in Eq. 9).

As stated above, the overall physical information available from the $F$ and $R$ realizations is not exploited either in Eq. 8 or in Eq. 9, To tackle this fact one can however apply a ML argument to the two collections of measurements implied by Eqs. 8 and 9 . We first notice that Eq. 8 has been derived maximizing the log function of the likelihood $\mathcal{L}\left(\Delta F_{A Q}\right)$

$$
\ln \mathcal{L}\left(\Delta F_{A Q}\right)=\sum_{i=1}^{n_{F}} \ln P\left(F \mid W\left[\mathbf{F}_{i}^{A q}\right]\right)+\sum_{j=1}^{n_{R}} \ln P^{\prime}\left(R \mid W\left[\mathbf{R}_{j}^{Q a}\right]\right) .
$$

Note that in Eq. 10 the probability in the second sum is primed. This means that such term must be treated with the usual reweighting procedure (see derivation of Eq. 8) because the realizations $\mathbf{R}^{Q a}$ are unavailable (since they must start from equilibrium microstates). The first sum of Eq. 10 can instead be treated in the standard fashion, because the work measurements $W\left[\mathbf{F}^{A q}\right]$ are available from the full $F$ realizations. Analogously, Eq. 9 has been obtained by maximizing the $\log$ function of $\mathcal{L}\left(\Delta F_{Q B}\right)$

$$
\ln \mathcal{L}\left(\Delta F_{Q B}\right)=\sum_{i=1}^{n_{F}} \ln P^{\prime}\left(F \mid W\left[\mathbf{F}_{i}^{Q b}\right]\right)+\sum_{j=1}^{n_{R}} \ln P\left(R \mid W\left[\mathbf{R}_{j}^{B q}\right]\right),
$$

where the probability in the first sum is primed for the same reasons discussed above. From a formal standpoint, Eqs. 10 and 11 deal with two independent collections of realizations, i.e., $\mathbf{F}^{A q}$ and $\mathbf{R}^{q a}$ the former equation and $\mathbf{F}^{q b}$ and $\mathbf{R}^{B q}$ the latter one. Therefore, noting that

$$
\Delta F_{Q B}=\Delta F_{A B}-\Delta F_{A Q}
$$

and assuming that the free energy difference $\Delta F_{A B}$ is known (using, e.g., the ML estimator of Eq. 2), we may express the overall likelihood of obtaining the given work measurements in both collections as the product $\mathcal{L}\left(\Delta F_{A Q}\right) \mathcal{L}\left(\Delta F_{Q B}\right)$, i.e., a function of $\Delta F_{A Q}$ alone (or alternatively, $\Delta F_{Q B}$ alone). Maximization of the log function of such a product with respect to $\triangle F_{A Q}$ leads to the following equation (the same estimator would be obtained maximizing the log function of $\mathcal{L}\left(\Delta F_{A Q}\right) \mathcal{L}\left(\Delta F_{Q B}\right)$ with respect to $\left.\Delta F_{Q B}\right)$

$$
\frac{\partial \ln \mathcal{L}\left(\Delta F_{A Q}\right)}{\partial \Delta F_{A Q}}+\frac{\partial \ln \mathcal{L}\left(\Delta F_{Q B}\right)}{\partial \Delta F_{A Q}}=0 .
$$


Since $\Delta F_{A B}$ is known, and hence independent on both $\Delta F_{A Q}$ and $\Delta F_{Q B}$, Eq. 12 sets the condition

$$
\frac{\partial \Delta F_{Q B}}{\partial \Delta F_{A Q}}=-1
$$

Using Eq. 14 into Eq. 13, leads to the relation

$$
\frac{\partial \ln \mathcal{L}\left(\Delta F_{A Q}\right)}{\partial \Delta F_{A Q}}-\frac{\partial \ln \mathcal{L}\left(\Delta F_{Q B}\right)}{\partial \Delta F_{Q B}}=0 .
$$

The left and right derivatives of Eq. [15 are exactly the left sides of Eqs. 8 and 9 , respectively. Therefore, upon substitution of Eqs. 8 and 9 into Eq. 15 and using Eq. 12 in the resulting equation, we obtain

$$
\begin{aligned}
& \sum_{i=1}^{n_{F}} \frac{1}{1+\frac{n_{F}}{n_{R}} \mathrm{e}^{\beta\left(W\left[\mathbf{F}_{i}^{A q}\right]-\Delta F_{A Q}\right)}}-\left\langle\mathrm{e}^{-\beta W\left[\mathbf{R}^{B q}\right]}\right\rangle^{-1} \sum_{j=1}^{n_{R}} \frac{\mathrm{e}^{-\beta W\left[\mathbf{R}_{j}^{B q}\right]}}{1+\frac{n_{R}}{n_{F}} \mathrm{e}^{\beta\left(W\left[\mathbf{R}_{j}^{q \alpha}\right]+\Delta F_{A Q}\right)}}- \\
& -\left\langle\mathrm{e}^{-\beta W\left[\mathbf{F}^{A q}\right]}\right\rangle^{-1} \sum_{k=1}^{n_{F}} \frac{\mathrm{e}^{-\beta W\left[\mathbf{F}_{k}^{A q}\right]}}{1+\frac{n_{F}}{n_{R}} \mathrm{e}^{\beta\left(W\left[\mathbf{F}_{k}^{q b}\right]-\Delta F_{A B}+\Delta F_{A Q}\right)}}+\sum_{l=1}^{n_{R}} \frac{1}{1+\frac{n_{R}}{n_{F}} \mathrm{e}^{\beta\left(W\left[\mathbf{R}_{l}^{B q}\right]+\Delta F_{A B}-\Delta F_{A Q}\right)}}=0 .
\end{aligned}
$$

Remember that in the equation above, the quantity $\Delta F_{A B}$ must be predetermined via Eq. 2. The left side of Eq. 16 is an increasing function in $\Delta F_{A Q}$ and the limits for $\Delta F_{A Q} \rightarrow \infty$ and for $\Delta F_{A Q} \rightarrow-\infty$ have opposite signs, being $n_{R}+n_{F}$ and $-n_{R}-n_{F}$, respectively. Again, this guarantees the existence of one unique root in the Eq. 16,

If from the one hand the ML estimator of Eq. 16 has the advantage of using the full physical information contained into our sets of work measurements, on the other hand it contains the free energy difference $\Delta F_{A B}$ that should be determined independently. This implies that the error on the estimate of $\Delta F_{A B}$ sums to that on $\Delta F_{A Q}$. The other derived ML estimators, i.e. Eqs. 8 and 9] do not suffer of such a shortcoming. The disadvantage is however that these ML estimators do not employ completely the physical information of the measurements in our hands.

\section{NUMERICAL TESTS: TECHNICAL DETAILS}

The ML estimators of Eqs. 8, 9 and 16 have been applied to compute the PMF for the unfolding process of the $\alpha$-helix form of an alanine deca-peptide $\left(\mathrm{A}_{10}\right)$ at finite temperature. Following Ref. 17, we have used steered MD simulations as a device for the numerical experiments, taking the end-to-end distance of $\mathrm{A}_{10}$ as reaction coordinate $\zeta$. In particular $\zeta$ corresponds to the distance between the $\mathrm{N}$ atom of the $\mathrm{N}$-terminus amino-acid (constrained to a fixed position) and the $\mathrm{N}$ atom of the C-terminus amino-acid (constrained to move along a fixed direction). The values of $\zeta$ in the folded and unfolded states of $\mathrm{A}_{10}$ are assumed[17] to be 15.5 and $31.5 \AA$, respectively. Moreover we have arbitrarily assumed the unfolding process of $\mathrm{A}_{10}$ as the forward $(F)$ one. In the context of our notation (see Sec. IIA), we therefore set $\zeta_{A}=15.5 \AA$ and $\zeta_{B}=31.5 \AA$. It should be noted that in general the end-to-end distance does not determine uniquely the configurational state of polypeptides. However, in the specific case of $\mathrm{A}_{10}$, the equilibrium distribution at $\zeta=\zeta_{A}$ corresponds to an ensemble of microstates tightly peaked around the $\alpha$-helix form, as for this end-to-end distance alternative structures are virtually impossible. The same holds true for the state corresponding to $\zeta=\zeta_{B}$, which basically represents an almost fully elongated configuration of the peptide. This implies that these two thermodynamic states can be effectively sampled using relatively few microstates obtained from equilibrium MD simulations at the given $\zeta$ values. The starting microstates for the $F$ and $R$ realizations have been randomly picked (every $5 \mathrm{ps)}$ ) from two standard MD simulations constraining $\zeta$ to $\zeta_{A}$ and to $\zeta_{B}$, respectively, by means of a stiff harmonic potential (force constant equal to $800 \mathrm{kcal} \mathrm{mol}^{-1} \AA^{-2}$ ). In both equilibrium MD simulations and in the subsequent steered MD simulations, constant temperature (300 K) has been enforced using a Nosé-Hoover thermostat [18, 19]. Force field has been taken from Ref. 20. Given the limited size of the sample, no cutoff radius has been imposed to the atomic pair interactions and no periodic boundary conditions have been applied.

For each type of process, $F$ and $R$, we have generated $10^{4}$ realizations guiding $\zeta$ from $\zeta_{A}$ to $\zeta_{B}$ ( $F$ realizations) or from $\zeta_{B}$ to $\zeta_{A}$ ( $R$ realizations) using a harmonic potential dependent on time:

$$
V(t)=\frac{k}{2}[\zeta-\lambda(t)]^{2},
$$

where the force constant $k$ is reported above. The time-dependence of the steering parameter $\lambda(t)$ determines the pulling speed of the nonequilibrium realizations and in general their time schedule. In our case, $\lambda(t)$ varies linearly with the time, i.e. $\lambda(t)=\zeta_{A}+\dot{\lambda} t$ for the $F$ realizations and $\lambda(t)=\zeta_{B}-\dot{\lambda} t$ for the $R$ ones. The work measurement 
at a given instant $t$ of a realization is calculated integrating the partial derivative of $V(t)$ with respect to time from the time zero to the time $t$. Six series of $R / F$ work measurements differing only in the pulling speed $\dot{\lambda}$ have been performed $\left(\dot{\lambda}=80,160,320,533,800\right.$, and $\left.1600 \AA \mathrm{ns}^{-1}\right)$.

\section{NUMERICAL TESTS: RESULTS}

In Fig. 2 we report a comparison between the PMF calculated using the ML estimators of Eqs. 8, 9 and 16 and the exact PMF recovered through thermodynamic integration[1]. To show the correctness of the estimators numerically,

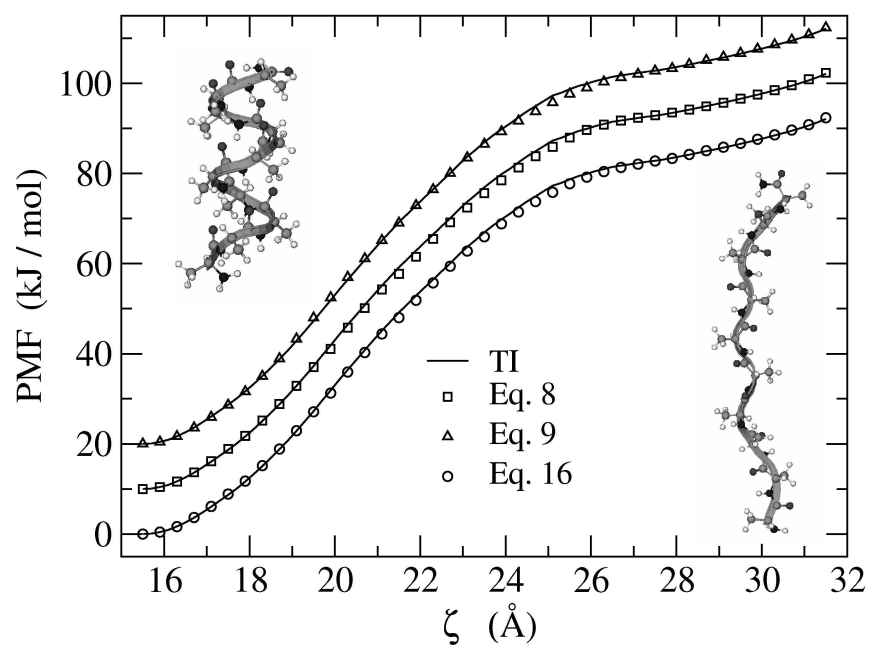

FIG. 2: PMF of $\mathrm{A}_{10}$ as a function of the reaction coordinate $\zeta$ (end-to-end distance). Squares: Eq. 8] triangles: Eq. 9] circles: Eq. [16 solid lines: thermodynamic integration (TI). The PMF profiles from ML estimators are calculated using $F$ and $R$ realizations performed with pulling speed of $80 \AA \mathrm{ns}^{-1}$. For the sake of clarity the curves are up-shifted.

in the figure we have drawn the PMF profiles obtained using the slowest pulling speed, i.e. $80 \AA \mathrm{ns}^{-1}$. It is noticeable the all ML estimators provide an almost perfect agreement with thermodynamic integration. The relevance of this result is enforced to the light of early PMF calculations 13] on the unfolding process of $\mathrm{A}_{10}$. Free energy estimators, such as Jarzynski equality and second order cumulant expansion [13], using only a slightly faster pulling speed (100 $\AA \mathrm{ns}^{-1}$ ), give a much worse accuracy than the methods proposed here. This can be more strictly verified comparing Fig. 5a of Ref. 13 with the PMF estimates determined using Eqs. 8 , 9 and 16 with pulling speed of $160 \AA \mathrm{ns}^{-1}$ (see supplementary material). A more comprehensive view of the performances of the ML estimators of Eqs. 8 , 9 and 16 is gained by the root mean square deviation of the estimated PMF curves from the exact one:

$$
\sigma=\left[\frac{1}{N} \sum_{i=1}^{N}\left(\mathcal{F}_{\mathrm{ML}}\left(\zeta_{i}\right)-\mathcal{F}_{\mathrm{TI}}\left(\zeta_{i}\right)\right)^{2}\right]^{\frac{1}{2}},
$$

where $\mathcal{F}_{\mathrm{ML}}\left(\zeta_{i}\right)$ is the value of the $\mathrm{PMF}$ at $\zeta=\zeta_{i}$ calculated using one of our ML estimators and $\mathcal{F}_{\mathrm{TI}}\left(\zeta_{i}\right)$ is the corresponding exact value determined by thermodynamic integration. In our calculations, the reaction coordinate is defined in steps of $0.4 \AA$, i.e. $\zeta_{1} \equiv \zeta_{A}=15.5, \zeta_{2}=15.9, \zeta_{3}=20.3, \cdots, \zeta_{N} \equiv \zeta_{B}=31.5 \AA$, where $N=41$. The value of $\sigma$ for the various approaches has been calculated after determining the additive constant of $\mathcal{F}_{\mathrm{ML}}(\zeta)$ via a least squares fitting to $\mathcal{F}_{\mathrm{TI}}(\zeta)$. The value of $\sigma$ obtained from the considered ML estimators for different pulling speeds is reported in Fig. 3. The worsening of the accuracy of the ML estimators by increasing the pulling speed of the realizations is expected on the basis of statistical reasons. The remarkable result is that all ML estimators have comparable accuracy independing on the pulling speed. Moreover, Eq. 9 gives the best accuracy for all pulling speeds except for $533 \AA \mathrm{ns}^{-1}$, while Eq. 16 gives systematically an accuracy which is in between those obtained from Eqs. 8 and 9. These facts suggest that the formal asymmetry of the ML estimators of Eqs. 8 and 9 (see discussion in Sec. IIC) in comparison to the formal symmetry of Eq. 16 may be relevant in the choice of the most accurate approach. In fact, it is known [15] that, for a given reaction path of a system, the use of a set of forward realizations in the framework of exponential averages for determining the free energy difference between two states may give different variance and bias with respect to the same estimate performed using the reverse realizations. We do not exclude that 


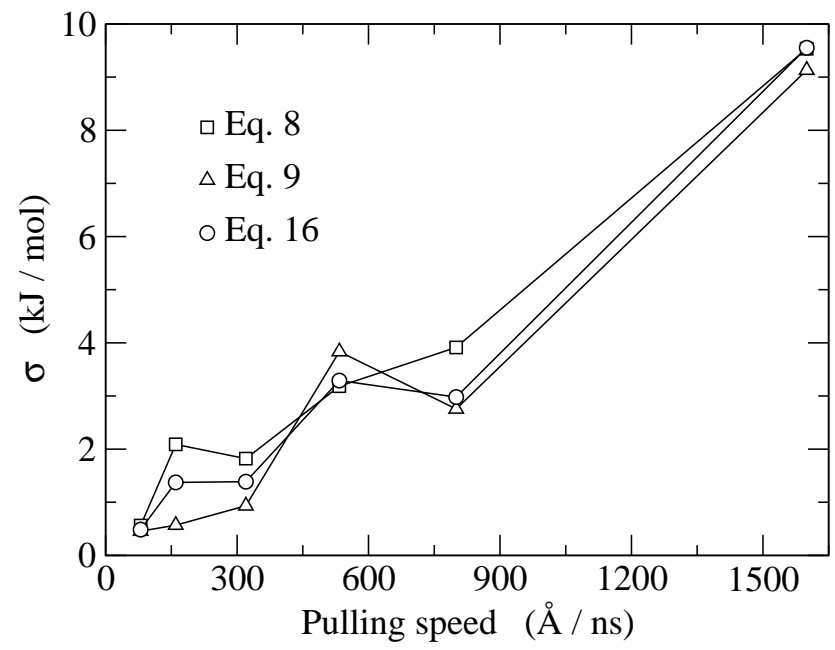

FIG. 3: $\sigma$ value (Eq. 18) as a function of the pulling speed for various ML estimators. Squares: Eq. 8 triangles: Eq. 9] circles: Eq. 16. The lines are drawn as a guide for eyes.

this fact might be related to our observations of Fig. 3 discussed above. In such a case the ML estimator of Eq. 16 would be the more appropriate without prior knowledge on the system.

\section{CONCLUSIONS}

We have presented a method for determining the PMF along a given reaction coordinate which is based on ML methods and path-ensemble averages in systems driven far from equilibrium. The method has been applied to computer experiments on the unfolding process of the $\alpha$-helix form of an alanine deca-peptide using nonequilibrium realizations with various pulling speeds. The estimated PMF is in fair agreement with thermodynamic integration. A formula for the variance of PMF estimates generated using this method is still unavailable, though its derivation appears straightforward following the guidelines reported in the present article and in the work by Shirts et al. 10 . We plan to report on this issue in a forthcoming contribution.

\section{Acknowledgments}

We thank David Minh (Department of Chemistry \& Biochemistry and Department of Pharmacology and NSF Center for Theoretical Biological Physics, University of California San Diego, USA) for providing insights about the generality of the ML estimators and for suggestions on how improve some parts of the manuscript. This work was supported by the European Union (Grant No. RII3-CT-2003-506350).

[1] J. G. Kirkwood, J. Chem. Phys. 3, 300 (1935).

[2] R. W. Zwanzig, J. Chem. Phys. 22, 1420 (1954).

[3] G. M. Torrie and J. P. Valleau, J. Comput. Phys. 23, 187 (1977).

[4] E. Darve and A. Pohorille, J. Chem. Phys. 115, 9169 (2001).

[5] A. Laio and M. Parrinello, Proc. Natl. Acad. Sci. USA 99, 12562 (2002).

[6] D. J. Evans, E. G. D. Cohen, and G. P. Morriss, Phys. Rev. Lett. 71, 2401 (1993).

[7] G. Gallavotti and E. G. D. Cohen, Phys. Rev. Lett. 74, 2694 (1995).

[8] C. Jarzynski, Phys. Rev. Lett. 78, 2690 (1997).

[9] G. E. Crooks, J. Stat. Phys. 90, 1481 (1998).

[10] M. R. Shirts, E. Bair, G. Hooker, and V. S. Pande, Phys. Rev. Lett. 91, 140601 (2003).

[11] C. H. Bennett, J. Comput. Phys. 22, 245 (1976).

[12] H. Oberhofer, C. Dellago, and P. L. Giessler, J. Phys. Chem. B 109, 6902 (2005).

[13] S. Park and K. Schulten, J. Chem. Phys. 120, 5946 (2004). 
[14] G. Hummer, J. Chem. Phys. 114, 7330 (2001).

[15] M. R. Shirts and V. S. Pande, J. Chem. Phys. 122, 144107 (2005).

[16] G. E. Crooks, Phys. Rev. E 61, 2361 (2000).

[17] P. Procacci, S. Marsili, A. Barducci, G. F. Signorini, and R. Chelli, J. Chem. Phys. 125, 164101 (2006).

[18] W. G. Hoover, Phys. Rev. A 31, 1695 (1985).

[19] W. G. Hoover, Phys. Rev. A 34, 2499 (1986).

[20] A. Mackerell, D. Bashford, M. Bellot, R. Dunbrack, J. Evanseck, M. Field, J. Gao, H. guo, S. Ha, D. Joseph-Mcarthy, et al., J. Phys. Chem. B 102, 3586 (1998).

[21] David Minh from Department of Chemistry \& Biochemistry and Department of Pharmacology and NSF Center for Theoretical Biological Physics, University of California San Diego, USA; private communication. 